Supporting information for

\title{
Predicting substrates by docking high-energy intermediates to enzyme structures
}

Johannes C. Hermann, Eman Ghanem, Yingchun Li, Frank M. Raushel, John J. Irwin

Brian K. Shoichet

Chart S1. Substrates for D-hydantoinase (HYD) contained in the docked databases.

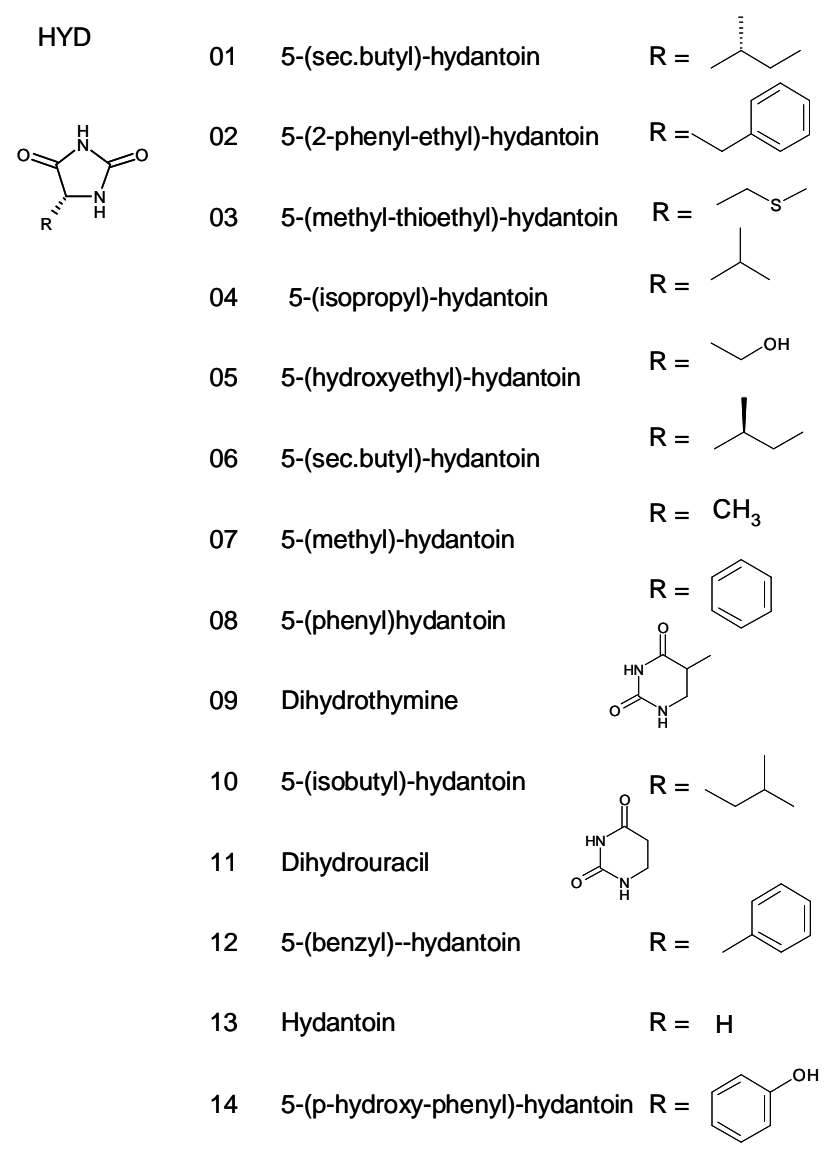


Chart S2. Substrates for phosphotriesterase (PTE) contained in the docked databases.

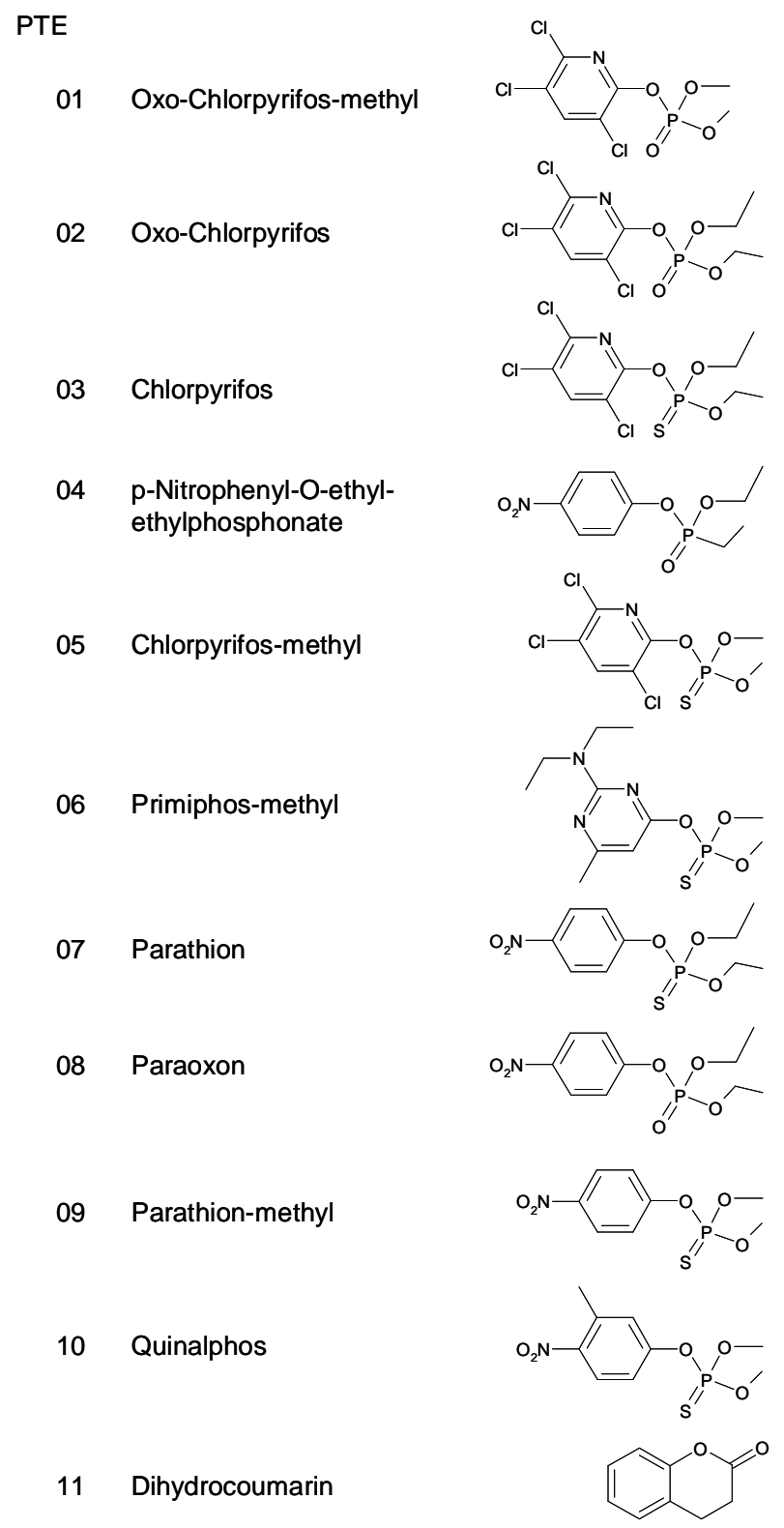


Chart S3. Substrates for N-acetyl-glucosamine-6-phosphate-deacetylase (NAGA), cytosine deaminase (CDA), dihydroorotate (DHO) and adenosine deaminase (ADA) contained in the docked databases.

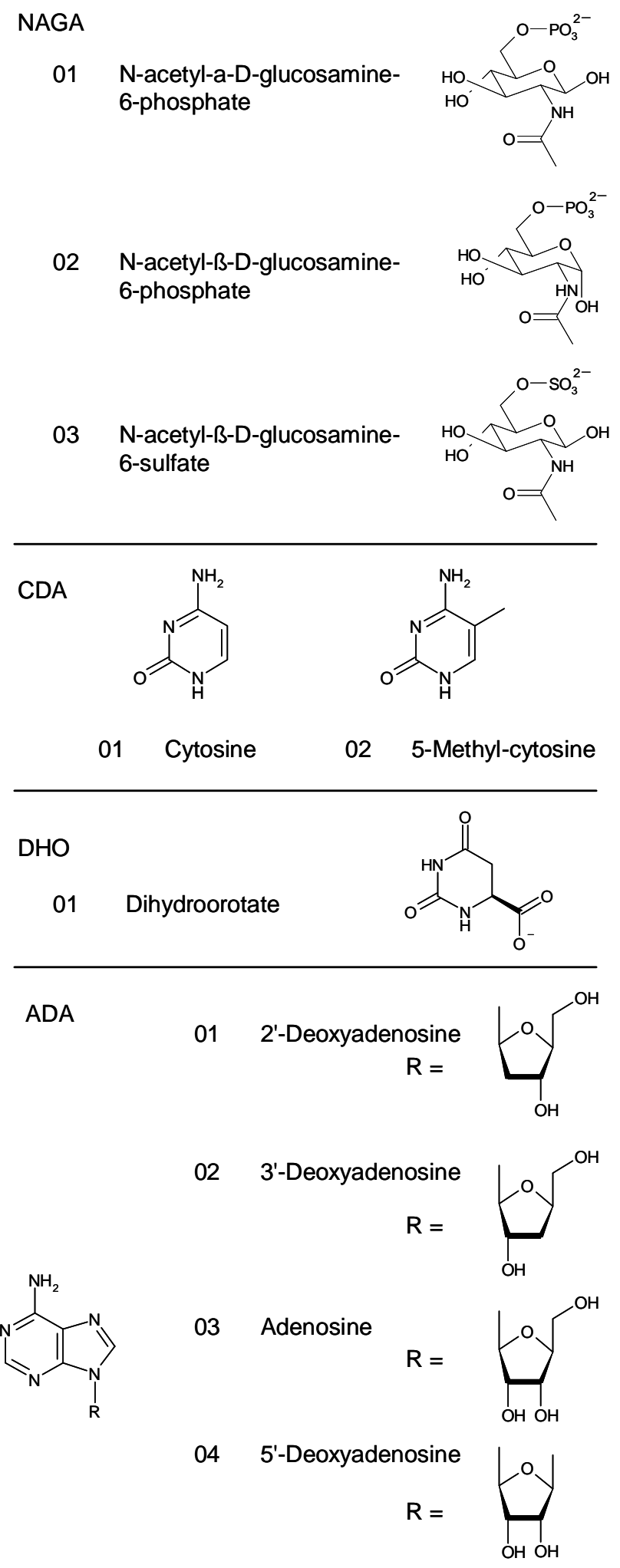

